\title{
Transient Pressure Behavior of a Well Located between a Constant Pressure Boundary and a Sealing Fault
}

\author{
R. Trabelsi, F. Boukadi, A. Seibi, D. Allen, F. Sebring, T. Mannon, H. Trabelsi \\ University of Louisiana, Lafayette, USA \\ Email: rfb2942@louisiana.edu
}

How to cite this paper: Trabelsi, R., Boukadi, F., Seibi, A., Allen, D., Sebring, F., Mannon, T. and Trabelsi, H. (2017) Transient Pressure Behavior of a Well Located between a Constant Pressure Boundary and a Sealing Fault. Natural Resources, 8, 646656. https://doi.org/10.4236/nr.2017.810041

Received: April 13, 2017

Accepted: October 28, 2017

Published: October 31, 2017

Copyright (c) 2017 by authors and Scientific Research Publishing Inc. This work is licensed under the Creative Commons Attribution International License (CC BY 4.0).

http://creativecommons.org/licenses/by/4.0/

\begin{abstract}
Pressure transient analysis has been used to evaluate performance of a well located between one sealing fault and one constant pressure boundary. Type curves were generated by determining 1) dimensionless pressure and 2) rate of change of dimensionless pressure drop with respect to dimensionless time. When the well is located closer to the no flow boundary, both sets of type curves have three distinct slopes. These slopes characterize: 1) flow in an infinite reservoir, 2) presence of the no flow, and 3) the constant-pressure boundaries. When the well is closer to the constant pressure boundary, the type curves show two distinct slopes. These correspond to: 1) flow in an infinite reservoir, and 2) the presence of a constant pressure boundary. The type curves can be used to match actual pressure drawdown data and determine the drainage area and relative well location with respect to physical boundaries.
\end{abstract}

\section{Keywords}

Pressure Transient, Principle of Superposition, Infinite Reservoir, No Flow Boundary, Constant Pressure Boundary, Drawdown, Drainage Area

\section{Introduction}

Pressure transient analysis has been widely used as an industry standard to determine reservoir boundaries. The boundaries present in reservoirs take the form of sealing and non-sealing faults. The identification of theses faults will enable engineers to determine the optimal production strategy of petroleum reservoirs, as well as provide insight on their long-term performance.

The detection of a linear fluid-barrier (fault) from pressure transient data was 
first presented by Horner [1]. Horner presented the foundation for pressure transient study of a single well located near a sealing fault. By applying the method of images, Horner was able to approximate the distance from a fault using pressure build up data.

This initial study sparked considerable investigation into the identification of petroleum reservoirs using pressure transient testing. Davis and Hawkins [2] identified the features which pressure transient curves must have to produce valid data. They then presented an equation to calculate relative distance to a fault using pressure drawdown data.

The theory of images was first applied to multiple boundary reservoirs by Jones [3]. In his study, Jones [3] used the reservoir limit test to analyze the performance of a gas well near two orthogonal sealing faults. After sufficient production, he noticed that the slope of the drawdown curve would become four times the initial value. This increase is created due to the reduction in production area, from an initial production of $360^{\circ}$ to a final production of $90^{\circ}$.

The pressure transient behavior of a well located near two intersecting boundaries was studied by Prasad [4]. Using the least squares method, he was able to determine many reservoir parameters including: relative distance to the fault, angle between the boundaries, flow capacity, and the initial reservoir pressure.

Tiab and Crichlow [5] developed a type-curve matching method for interpreting pressure transient curves of a well located inside multiple sealing faults and rectangular reservoir systems. This study was extended when Tiab and $\mathrm{Ku}-$ mar [6] investigated the pressure transient behavior of a constant producing well located between two parallel sealing faults. In their paper, they outlined several different techniques to both detect and locate these faults.

Previous studies have indicated that sealing faults yield to deviation between a well's pressure response and the homogeneous line-source response. Brit to and Grader [7] determined that pressure response is extremely sensitive to the size, shape, and orientation of the impermeable region.

\section{Dimensionless Diffusivity Equation}

The dimensionless linear diffusivity equation can be used to generate pressures at distinct reservoir locations and times. The linear diffusivity equation is given by the equation shown below:

$$
p_{D}\left(r_{D}, t_{D}\right)=-\frac{1}{2} E i\left(-\frac{r_{D}^{2}}{4 t_{D}}\right)
$$

where $r_{D}=\frac{r}{r_{W}}, t_{D}=\frac{0.000264 k t}{\phi \mu c r_{w}^{2}}, p_{D}=\frac{2 \pi h k}{q B \mu}\left[p_{i}-p(r, t)\right]=q_{D}\left[p_{i}-p(r, t)\right]$.

Upon setting $\alpha=\frac{r_{D}^{2}}{4 t_{D}}$, the dimensionless diffusivity equation becomes:

$$
p_{D}\left(r_{D}, t_{D}\right)=-\frac{1}{2} \operatorname{Ei}(-\alpha)
$$


The $E i$ in the above equation is known as the Exponential Integral and is defined as either

$$
\operatorname{Ei}(\alpha)=\int_{-\infty}^{\alpha} \frac{\mathrm{e}^{-s}}{s} \text { or }-E i(-\alpha)=\int_{\alpha}^{-\infty} \frac{\mathrm{e}^{-s}}{s}
$$

Or,

$$
-E i(-\alpha)=\int_{\alpha}^{\infty} \frac{\mathrm{e}^{-s}}{s} \mathrm{~d} s \approx(-\ln (\alpha)-\gamma)+\left[\alpha-\frac{\alpha^{2}}{2 \times 2 !}+\frac{\alpha^{3}}{3 \times 3 !}-\cdots\right]
$$

where $\gamma \approx 0.5772157$ is Euler's Constant and $\left[\alpha-\frac{\alpha^{2}}{2 \times 2 !}+\frac{\alpha^{3}}{3 \times 3 !}-\cdots\right]$ is the error associated with the Ei formula. Assuming the error to be negligible, Equation (4) can be reduced to the following:

$$
-E i(-\alpha)=\int_{\alpha}^{\infty} \frac{\mathrm{e}^{-s}}{s} \mathrm{~d} s \approx(-\ln (\alpha)-0.5772157)
$$

Substituting this equation into the linear diffusivity equation yields:

$$
p_{D}\left(r_{D}, t_{D}\right)=-\frac{1}{2}\left(\ln \frac{r_{D}^{2}}{4 t_{D}}+0.5772157\right)
$$

The properties of natural logs can then be used for further simplification. The resulting equation becomes:

$$
p_{D}\left(r_{D}, t_{D}\right)=\frac{1}{2}\left(\ln \left(t_{D}\right)-\ln \left(r_{D}^{2}\right)+0.80908\right)
$$

when evaluating the wellbore pressure, it is assumed that $r=r_{w}$. In making this assumption, $r_{D}=\frac{r}{r_{w}}=\frac{r_{w}}{r_{w}}=1$. Substituting this value into the previous equation, yields the following result:

$$
p_{D}\left(r_{D}=1, t_{D}\right)=\frac{1}{2}\left(\ln \left(t_{D}\right)+0.80908\right)
$$

\section{Superposition Principle}

The superposition principle has been used by petroleum engineers to solve complex problems for many decades. The superposition principle states that the response of a given system caused by two or more stimuli is the sum of the responses of each individual stimulus. Superposition, as well as the theory of images, can be used to model reservoirs with single or multiple boundaries. Superposition can be used to generate solution to two different scenarios: 1) superposition in space, and 2) superposition in time (Lee, Rollins, and Spivey [8]).

\section{Two-Parallel Fault System}

Consider a single well producing at a constant rate between two-parallel boundaries. Unlike Tiab and Kumar [6], who studied well behavior between two sealing faults, the goal of this research was to identify well performance between one sealing fault and one constant pressure boundary. 
Using the Principle of Superposition, the pressure transient behavior of this system can be easily generated. To determine the dimensionless pressure equation, it is integral to define the real and imaginary wells present in the system. Assume that our system follows the illustration shown in Figure 1.

By applying the Principle of Superposition, we can define the dimensionless pressure of Well A as the sum of pressure contributions from the real well and the four imaginary wells. The dimensionless pressure equation can be expressed as:

$$
p_{D}\left(r_{D}, t_{D}\right)=p_{D}\left(r_{D}=1, t_{D}\right)+\sum_{i=1}^{4} p_{D}\left(r_{D i}, t_{D}\right)
$$

where, $p_{D}\left(r_{D}=1, t_{D}\right)=\Delta P$ created by well $\mathrm{A}$, and $\sum_{i=1}^{4} p_{D}\left(r_{D i}, t_{D}\right)=\Delta P$ created by the four imaginary wells. Using Equations (1) \& (8) the dimensionless pressure equation becomes:

We can then simplify this equation to generate:

$$
\begin{aligned}
p_{D}\left(r_{D}, t_{D}\right)= & {\left[\frac{1}{2}\left(\ln \left(t_{D}\right)+0.80908\right)\right]+\left[-\frac{1}{2} E i\left(-\frac{(2 a)^{2}}{4 t_{D}}\right)\right]+\left[-\frac{1}{2} E i\left(-\frac{(2 b)^{2}}{4 t_{D}}\right)\right] } \\
& +\left[-\frac{1}{2} E i\left(-\frac{(2 a+2 b)^{2}}{4 t_{D}}\right)\right]+\left[-\frac{1}{2} E i\left(-\frac{(2 a+2 b)^{2}}{4 t_{D}}\right)\right]
\end{aligned}
$$

We can then simplify this equation to generate:

$$
\begin{aligned}
p_{D}\left(r_{D}, t_{D}\right)= & {\left[\frac{1}{2}\left(\ln \left(t_{D}\right)+0.80908\right)\right]+\left[-\frac{1}{2} E i\left(-\frac{a^{2}}{t_{D}}\right)\right]+\left[-\frac{1}{2} E i\left(-\frac{b^{2}}{t_{D}}\right)\right] } \\
& +\left[-E i\left(-\frac{(a+b)^{2}}{t_{D}}\right)\right]
\end{aligned}
$$

The dimensionless pressure equation must then be corrected for the individual nature of the imaginary wells. Since producer wells have positive production while injector wells have negative production, the pressure contribution of injector wells must be multiplied by -1 . The dimensionless pressure equation then becomes:

$$
\begin{aligned}
p_{D}\left(r_{D}, t_{D}\right)= & {\left[\frac{1}{2}\left(\ln \left(t_{D}\right)+0.80908\right)\right]-\left[\frac{1}{2} E i\left(-\frac{a^{2}}{t_{D}}\right)\right] } \\
& +\left[\frac{1}{2} E i\left(-\frac{b^{2}}{t_{D}}\right)\right]+\left[E i\left(-\frac{(a+b)^{2}}{t_{D}}\right)\right]
\end{aligned}
$$

\section{Dimensionless Pressure Type Curve Generation}

Type curves were generated by determining the dimensionless pressure drop with respect to changes in dimensionless times. Several type curve iterations were constructed by altering (1) the distance between boundaries, and (2) the relative location of the well.

Reservoir size was evaluated at four intervals: 10, 100, 1000, and 10,000 ft. For each reservoir, the "real" production well was then positioned at nine distinct 


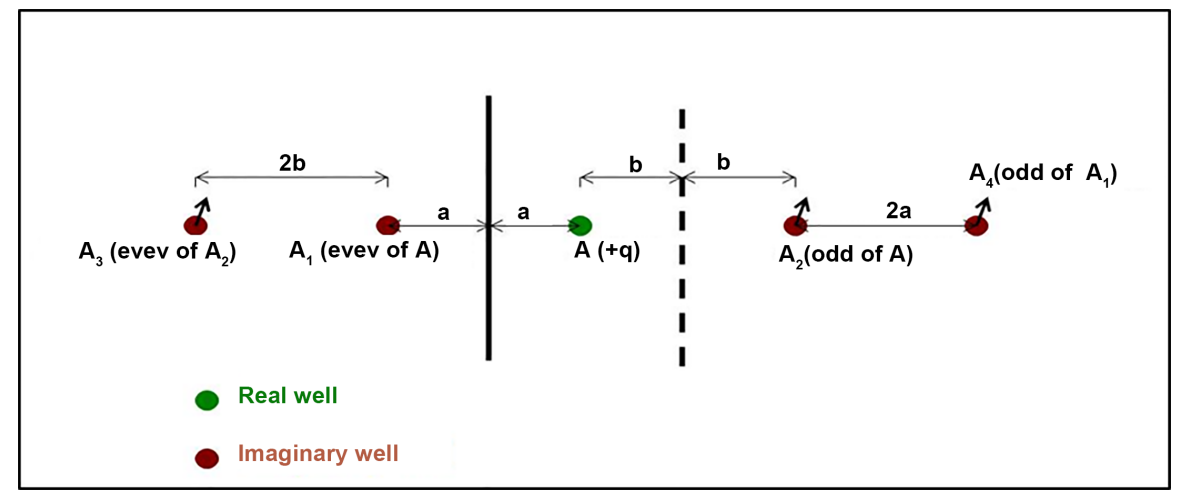

Figure 1. Location of a well between two distinct boundaries.

locations: $10 \%, 20 \%, 30 \%, 40 \%, 50 \%, 60 \%, 70 \%, 80 \%$, and $90 \%$ from the no flow boundary with respect to total distance between the two boundaries. To better understand this terminology, please refer to Figure 2.

The four dimensionless pressure type curves are shown in Figures 3-6.

In each figure, the initial slope corresponds to well behavior in an infinite reservoir. This flow regime is commonly known as transient flow. After a certain dimensionless time, the reservoir will no longer behave as if it had infinite size; this process is marked by a deviation in the initial straight line. This deviation marks the detection of either reservoir boundary. Several factors affect the time at which a well remains in an infinite system. These are 1) total distance between the boundaries, 2) the "real" well's distance to the boundaries, and 3) the identity of the boundaries.

For this study, a "real" well was placed between a sealing fault and constant pressure boundary. As distance between the two boundaries increase, the production well will display transient flow behavior for a longer period of time.

After the "real" well senses a boundary, it begins to deviate from the initial straight line. When the production well is closer to the no flow boundary than the constant pressure boundary $(10 \%, 20 \%, 30 \%$, and $40 \%)$, a second slope will occur, which indicates the presence of a no flow boundary.

The slope of the second straight line is a direct function of the well's distance from the no flow boundary. As the location of the "real" well moves closer to the no flow boundary, it becomes affected by this boundary for both a longer period of time and greater magnitude, prior to being influenced by the constant pressure boundary. Thus, the slope of the second straight line will increase from 1.15 to 1.5 times that of the initial straight line, as distance to the no flow boundary decreases. This occurs because the cone of depression from production has encountered a volume confining barrier. At this point, the reservoir extent has been restricted on one side of the well's production zone while production rate has remained constant. Now that production volume has been limited, the rate of pressure drop will be greater than it was in the initial transient condition, as it is now producing from a confined system rather than an infinite acting system.

After a certain period of time, a second deviation will occur, which marks the effect of the constant pressure boundary. This deviation will ultimately lead to a 


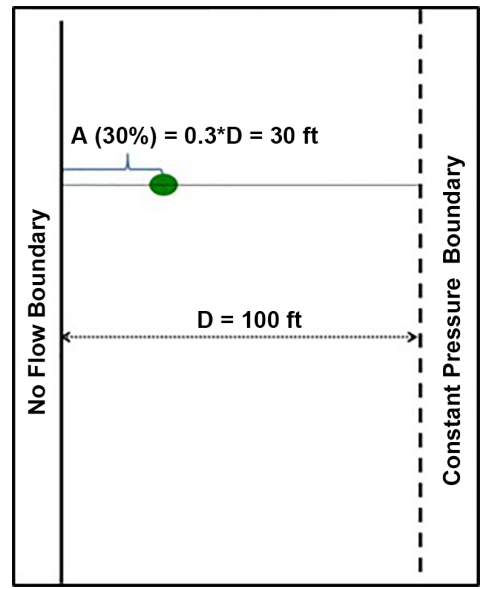

Figure 2. Representation of boundary distance.

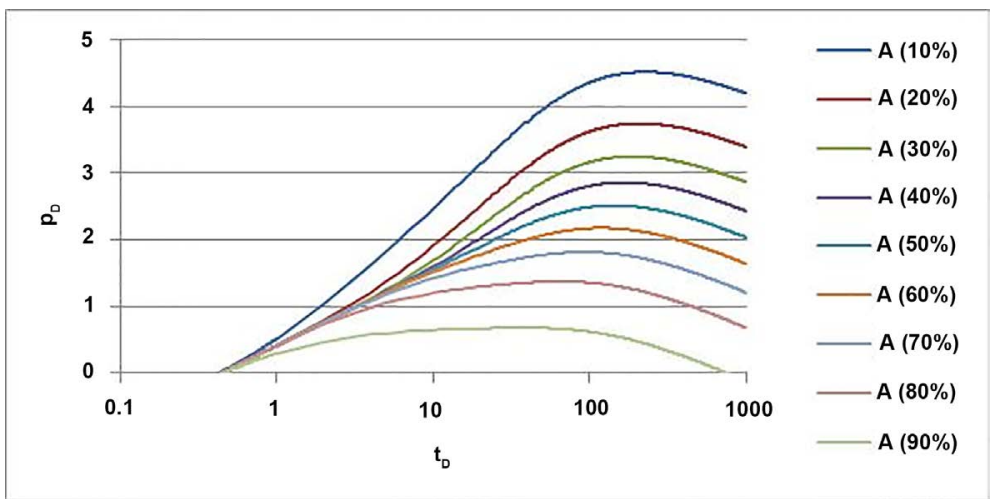

Figure 3. Dimensionless pressure vs. dimensionless time $-\mathrm{D}=10 \mathrm{ft}$.

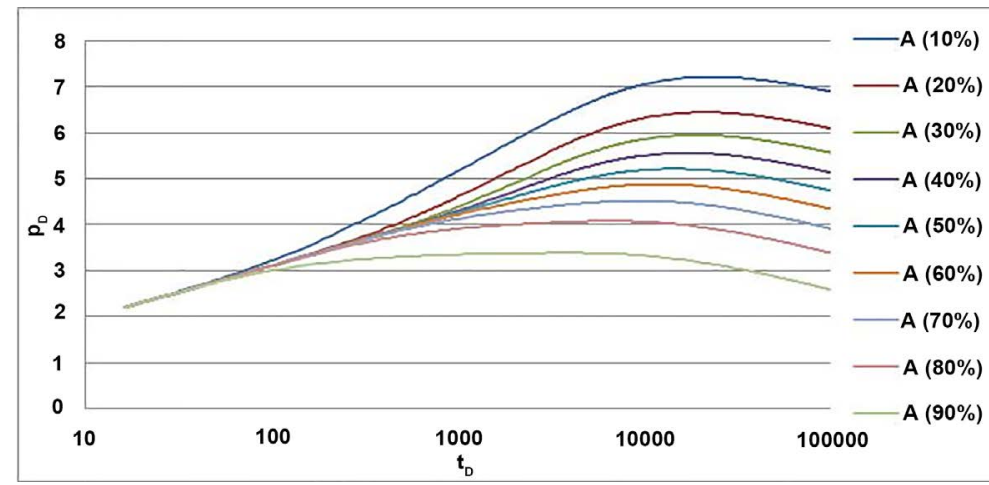

Figure 4. Dimensionless pressure vs. dimensionless time $-\mathrm{D}=100 \mathrm{ft}$.

downward sloping straight line, which is always the opposite slope of the initial straight line, despite the well's location in the reservoir. The opposite slope is due to the fact that rate of reservoir replenishment is equal to rate of fluid removal. As the "real" well moves closer to the constant pressure boundary, the slope will occur at an earlier dimensionless time since volume replenishment will take place sooner.

Since flow rates are assumed constant, volume replenishment will be sensed at an earlier time for wells located closer to the constant pressure boundary. This 


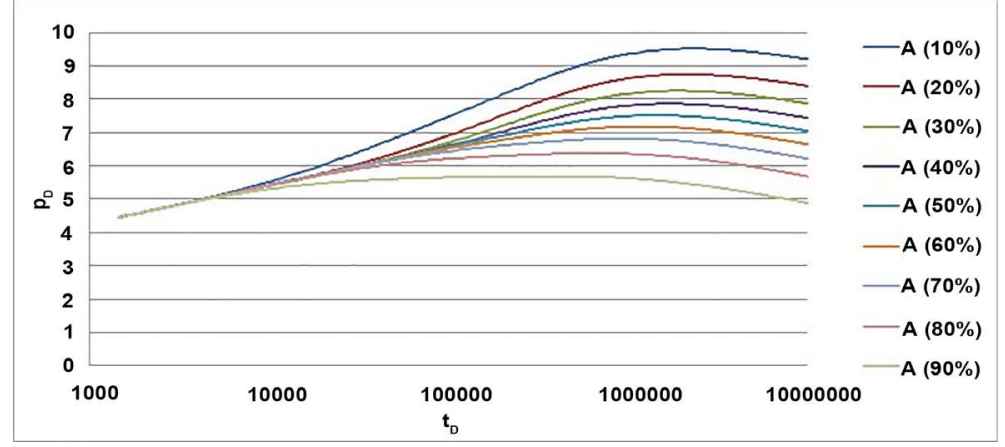

Figure 5. Dimensionless pressure vs. dimensionless time $-\mathrm{D}=1000 \mathrm{ft}$.

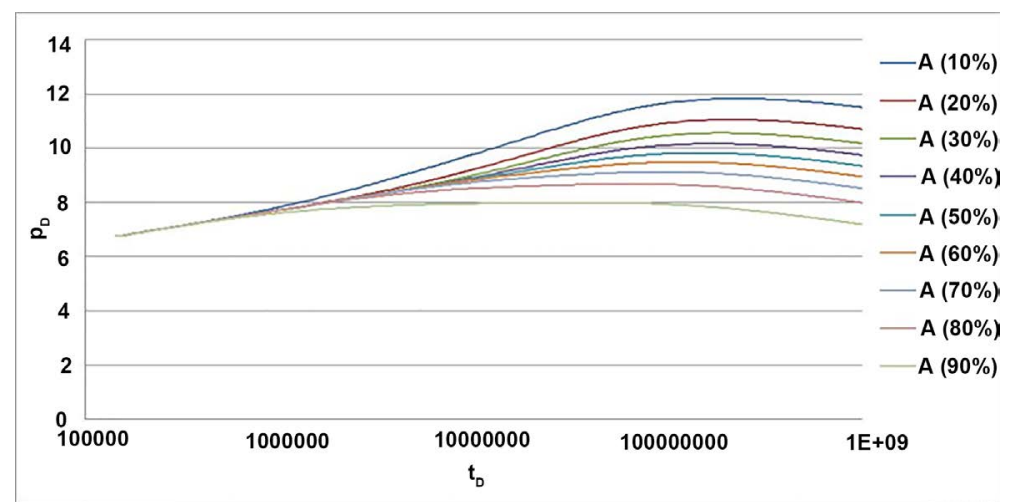

Figure 6. Dimensionless pressure vs. dimensionless time $-\mathrm{D}=10,000 \mathrm{ft}$.

replenishment will lessen the increase in pressure drop rate associated with the no flow boundary. Therefore, the slope the second deviation will be reduced.

\section{Rate of Change of Dimensionless Pressure}

The time rate of change of dimensionless pressure is obtained by differentiating Equation (12) with respect to dimensionless time. Thus,

$$
p_{D}^{\prime}\left(r_{D}, t_{D}\right)=p_{D}\left(r_{D}, t_{D}\right) \frac{\mathrm{d} p_{D}}{\mathrm{~d} t_{D}}
$$

Substituting the original dimensionless pressure equation into the equation yields:

$$
\begin{aligned}
p_{D}^{\prime}\left(r_{D}, t_{D}\right)= & {\left[\left[\frac{1}{2}\left(\ln \left(t_{D}\right)+0.80908\right)\right]-\left[\frac{1}{2} E i\left(-\frac{a^{2}}{t_{D}}\right)\right]\right.} \\
& \left.+\left[\frac{1}{2} E i\left(-\frac{b^{2}}{t_{D}}\right)\right]+\left[E i\left(-\frac{(a+b)^{2}}{t_{D}}\right)\right]\right] \frac{\mathrm{d} p_{D}}{\mathrm{~d} t_{D}}
\end{aligned}
$$

Taking the derivative of each term on the right side of the equation 14 allows us to compute the derivative of the entire equation. Upon differentiation, the resulting formula is:

$$
p_{D}^{\prime}\left(r_{D}, t_{D}\right)=\frac{1}{2 t_{D}}+\left(\frac{1}{2 t_{D}}\right) \mathrm{e}^{\frac{-a^{2}}{t_{D}}}-\left(\frac{1}{2 t_{D}}\right) \mathrm{e}^{\frac{-b^{2}}{t_{D}}}-\left(\frac{1}{t_{D}}\right) \mathrm{e}^{\frac{-(a+b)^{2}}{t_{D}}}
$$


Type curves were generated by determining the time rate of change of dimensionless pressure with respect to changes in dimensionless times. As with the previous section, type curves were created by altering (1) the distance between boundaries, and (2) the relative location of the well.

Reservoir size was evaluated at four intervals: 10, 100, 1000, and 10,000 ft. For each reservoir, the "real" production well was then evaluated at nine distinct locations: $10 \%, 20 \%, 30 \%, 40 \%, 50 \%, 60 \%, 70 \%, 80 \%$, and $90 \%$ from the no flow boundary (with respect to total distance between boundaries).

The four dimensionless pressure type curves are shown in Figures 7-10.

In each figure, there are two distinct types of curves based upon the relative location of the "real" well. As in the previous section, the first slope identifies well behavior when it is closer to the no flow boundary, while the second slope identifies well behavior when it is closer to the constant pressure boundary.

Despite the location of the well, both curves begin with a straight line portion corresponding to the well in an infinite reservoir. When the well is located closer to the no flow boundary, we observe two more distinct linear portions. The second linear portion corresponds to the effects of a closer, no flow boundary. As in the previous example, the confining volume increases the rate of dimensionless pressure drop, which leads to an acceleration of dimensionless pressure drop, shown in Figures 7-10.

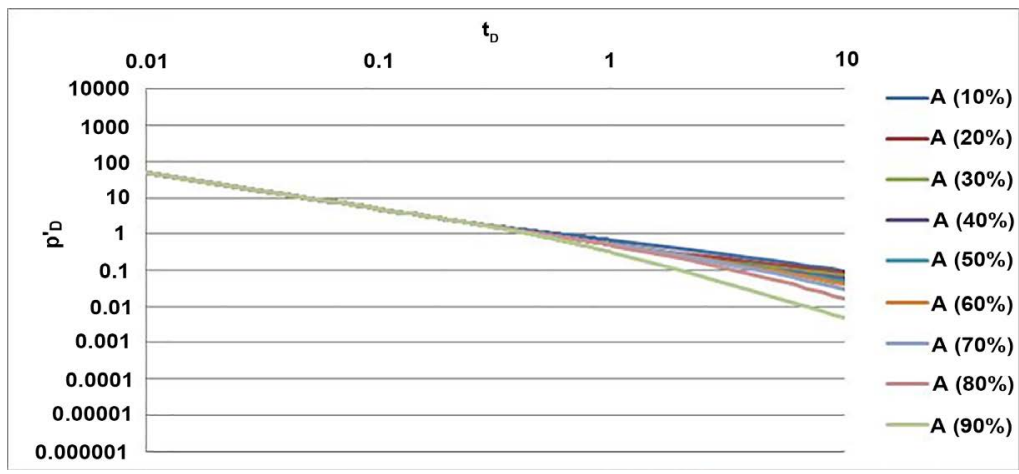

Figure 7. Rate of change of dimensionless pressure vs. dimensionless time-D $=10 \mathrm{ft}$.

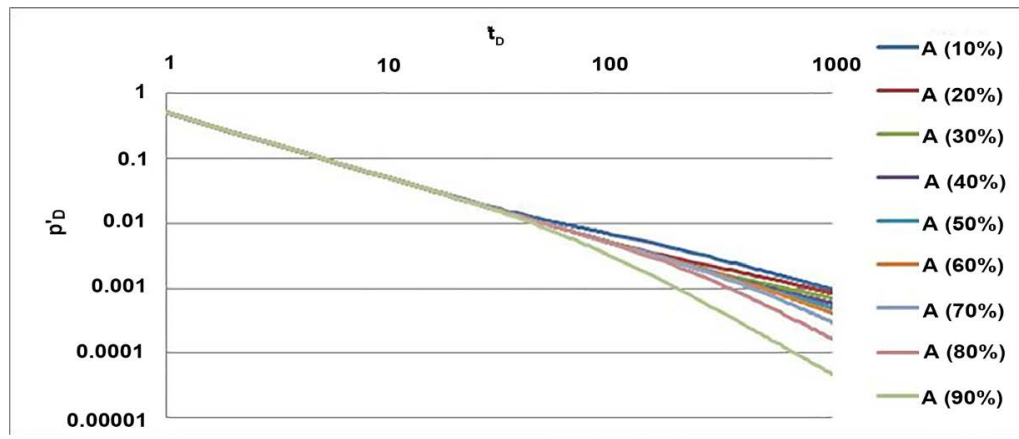

Figure 8. Rate of change of dimensionless pressure vs. dimensionless time-D $=100 \mathrm{ft}$. 


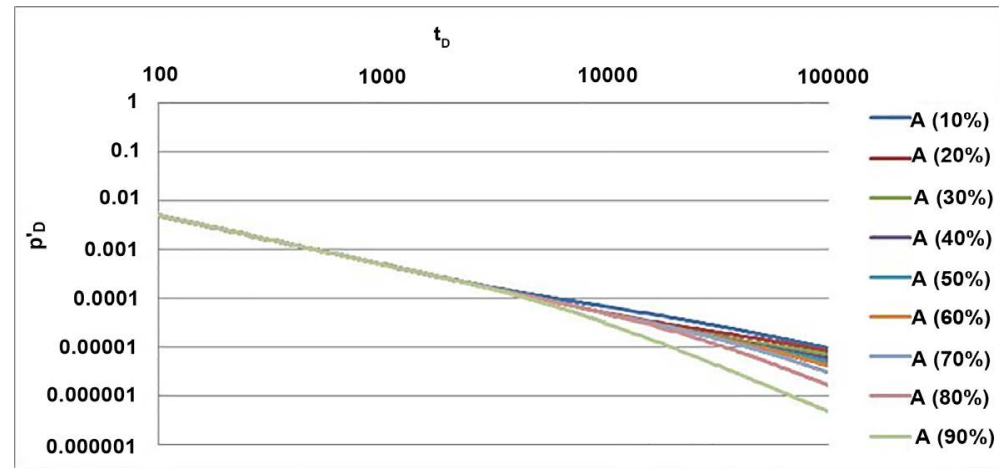

Figure 9. Rate of change of dimensionless pressure vs. dimensionless time$\mathrm{D}=1000 \mathrm{ft}$.

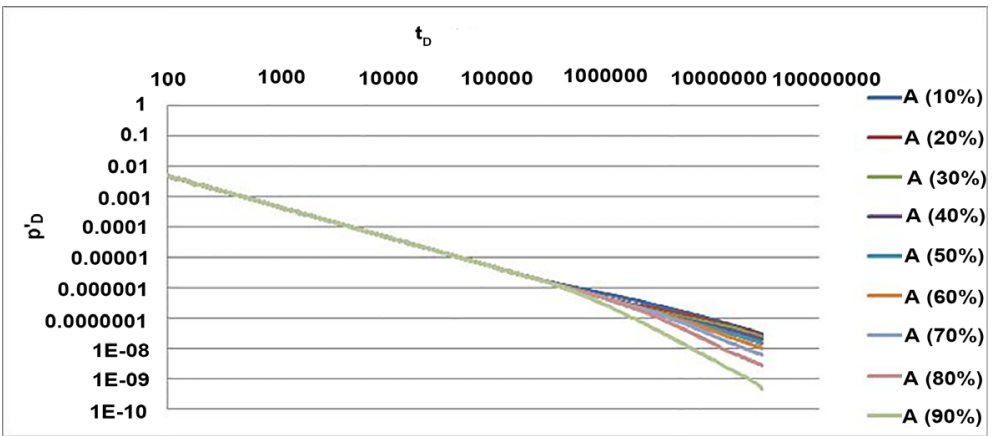

Figure 10. Rate of change of dimensionless pressure vs. dimensionless time-D $=10,000 \mathrm{ft}$.

As the well moves farther from the no flow boundary, the slope change reduces in magnitude, signifying a reduction in the acceleration of dimensionless pressure drop. At a certain dimensionless time, the well will sense the constant pressure boundary. This point will be marked by a deviation of the second slope, ultimately yielding the formation of a third slope. Over time, we can evaluate the slope of this line to be roughly twice that of the original straight line. This change in slope, as mentioned in the previous section, occurs because voidage replacement takes place in the reservoir, and rate of dimensionless pressure drop decreases. This decrease in dimensionless pressure drop over a given time period results in deceleration of dimensionless pressure drop, as observed in Figures 7-10.

When the well is located closer to the constant pressure boundary, it follows that only two straight line portions exist. As explained above, the first straight line corresponds to a well in an infinite reservoir. The second slope represents the effect of the constant pressure boundary, hence, reservoir replenishment. Since flow rates are constant for all wells, this slope will be roughly twice as steep as the initial trend.

\section{Conclusions}

The goal of this study was to characterize the behavior of a well situated between 
a sealing fault and constant pressure boundary. Using the principle of superposition, we were able to define the system using a "real" well and a series of imaginary wells, which served as the boundary effects. After the system was identified, the diffusivity equation was developed using contributions from each well (both real and imaginary) as well as the exponential integral (Ei) function. Type curves were then generated to evaluate 1) dimensionless pressure drop and 2) rate of change of dimensionless pressure drop, as functions of dimensionless time. For both varieties of type curves, the following results were identified:

1) For A (10\%) to A (40\%), three distinct slopes were visible.

a) $1^{\text {st }}$ Slope-Signifies flow in an infinite reservoir;

b) $2^{\text {nd }}$ Slope-Identifies the presence of the no flow boundary;

c) $3^{\text {rd }}$ Slope-Corresponds to the presence of a constant pressure boundary.

2) As the real well moves closer to the sealing fault, it was affected in a greater magnitude by the no flow boundary. Thus, an increase in pressure drop was evident.

3) For A (60\%) to A (90\%), two distinct lines were visible.

a) $1^{\text {st }}$ Slope-Signifies flow in an infinite reservoir;

b) $2^{\text {nd }}$ Slope-Corresponds to the presence of a constant pressure boundary.

4) As the "real" well becomes situated closer to the constant pressure boundary, the second slope is formed at an earlier time.

5) A difference between the two type curve sets occur with the slope of the last line.

a) Dimensionless Pressure Drop-Last slope opposite of the initial slope;

b) Rate of change of dimensionless pressure drop-Last slope roughly twice as steep as initial slope.

\section{References}

[1] Horner, D.R. (1951) Pressure Build-Up in Wells. Proceedings of the 3rd World Petroleum Congress, 25-43.

[2] Davis, E.G. and Hawkins, M.F. (1963) Linear Fluid-Barrier Detection by Well Pressure Measurements. Journal of Petroleum Technology, 15, 1077-1081. https://doi.org/10.2118/462-PA

[3] Jones, P. (1962) Reservoir Limit Test on Gas Wells. Journal of Petroleum Technology, 14, 613-619. https://doi.org/10.2118/24-PA

[4] Prasad, R.K. (1975) Pressure Transient Analysis in the Presence of Two Intersecting Boundaries. Journal of Petroleum Technology, 27, 89-96. https://doi.org/10.2118/4560-PA

[5] Tiab, D. and Crichlow, H.B. (1979) Pressure Analysis of Multiple-Sealing-Fault Systems and Bounded Reservoirs by Type-Curve Matching. Journal of Petroleum Technology, 19, 378-392. https://doi.org/10.2118/6755-PA

[6] Tiab, D. and Kumar, A. (1980) Detection and Location of Two Parallel Sealing Faults around aWell. Journal of Petroleum Technology, 20, 1701-1708.

[7] Britto, P.R. and Grader, A.S. (1988) The Effects of Size, Shape, and Orientation of an Impermeable Region on Transient Pressure Testing. SPE Formation Evaluation, 3, 595-606. https://doi.org/10.2118/16376-PA 
[8] Lee, J., Rollins, J.B. and Spivey, J.P. (2003) Pressure Transient Testing. Society of Petroleum Engineers Textbook Series, Vol. 9, Richardson.

\section{Nomenclature}

$P_{D}=$ Dimensionless Pressure

$r_{D}=$ Dimensionless Radius

$t_{D}=$ Dimensionless Time

$q_{D}=$ Dimensionless Production Rate

$r=$ Distance from Well $(\mathrm{ft})$

$r_{w}=$ Wellbore Radius $(\mathrm{ft})$

$k=$ Reservoir Permeability $(\mathrm{mD})$

$t=$ Time of Production (hrs)

$\phi=$ Reservoir Porosity

$\mu=$ Fluid Viscosity (cp)

$c=$ Total Formation Compressibility $\left(\mathrm{psi}^{-1}\right)$

$q=$ Production Rate (stb/day)

$B=$ Formation Volume Factor ( $\mathrm{rbbl} / \mathrm{stb}$ )

$h=$ Average Reservoir Thickness (ft)

$p_{i}=$ Initial Reservoir Pressure (psi) 